\title{
ON FALLING.
}

BY BAMUEL WILKS, M.D., LL.D., F.R.8.

Amongst the various symptoms which a medical man is called upon to interpret, there is none more important than that of sudden falling. He usually endeavours to distinguish between an epilepsy and syncope, although these two conditions by no means cover all the possible causes for the occurrence; if it has taken place without loss of consciousness, he has to seek much further for its antecedents. He has therefore to discuss with himself some of the most difficult problems connected with the working of the nervous system, although fully aware that no perfect interpretation can be made of his case until these have been solved by the physiologist. Nevertheless he makes use of the knowledge which he possesses, to guide him as far as possible towards the light. One of the most important facts which the clinical observer has of late gained from a study of disease has been independently worked out in the laboratory, viz. the much more intimate connection between the nerve-centres, nerve-fibres and muscles, than was ever before conceived. A nerve now is looked upon as the actual process of a cell, and its termination in end plates as closely incorporated with the muscle. No longer is a grey centre regarded as a mere store-house of electricity; the nerve a telegraphic wire; and the muscle a receptacle of force, like a packet of nitro-glycerine, ready to be exploded by the pull of a string, but the very nutrition of the muscle and its capability of storing up energy is known to be dependent on the nerves to which it is attached. It is obvious, therefore, that all changes in the motor centres will be exhibited by some corresponding alteration in the muscles. This has. become a truism, so that the diagnosis of a central lesion is made when no other indication of it exists than a weakened state of some set of muscles. We daily speak of the blocking 
of an artery in the brain, or the rupture of a cerebral vessel, when not a single characteristic symptom of disease of the organ is present; we judge only by the fact of certain muscles associated through the nerves and spinal cord with a particular part of the brain being paralysed. This close union of nerve activity and muscle movement is recognised through the whole animal kingdom. The position and attitude of an animal testify to the working of the brain within; whether it holds itself ready to pounce on its prey, or lie crouching with fear.

In man the attitude of defiance, with well-knit muscles, and that of the trembling coward, exhibits at once the close connection between the muscles and the nervous system. The different positions of the hands and limbs as indicative of the ever-varying passions of the mind have been fully illustrated by Dr. Warner in his work on "Physical Expressions." The hard physiognomy of Julius Cæsar and the more supple features of Homer are seen in striking contrast in their respective busts in the British Museum.

The close correspondence between the nerve-centres and muscles is well exemplified in the exhibition of the so-called "thought reading." Not only does the nerve-centre stimulate the muscle to action, but the mere thought of a movement will influence it, as if the motor region of the brain could not in mere imagination be employed (if we may use so roundabout an expression) without a corresponding effect on those muscles which would be engaged in the movement; so that if the subject of the experiment be told to fix his attention closely upon the thing which he has hidden, the muscles which would have taken him to the place of concealment undergo a tension which is quite capable of being appreciated by any one who chooses to cultivate by practice this manipulative art. It is the unconsciousness of this change in his muscles which makes the subject of the experiment believe that the operation is a purely mental one. I apprehend that this is a law of our nature, and that in various processes of thought the different muscles which would outwardly express them are put into action, so that some persons with little power of restraint think aloud. I have been more than once struck with this when driving along the streets on the occasion of some damage to 
one's carriage, or of one's horse having gone lame; the common or uneducated people will openly proclaim the fuct. It no doubt has also passed through the brain of every one on the road, but the better educated inhibited the outward expression of it. I mention in my published Lectures the case of a singer, who, when reading a piece of music mentally, "hums" the air; but if he has a sore throat, or is hoarse, he cannot appreciate its merits in the same degree as when he is well.

Mr. Francis Galton ${ }^{1}$ made some very interesting observations, according to his usual scientific and rigorous method, by which he measured the boredom of an audience by the tone of their muscles. If the discourse was dry, the persons lolled and held the most varying inclinations towards one another; but as soon as the speaker said anything interesting, the audience braced themselves úp, presented a fixed attitude, so as to become equidistant from one another. Mr. Gralton thinks by a little trouble he could frame an exact measurement of the intentness or indifference of an audience.

It is evident, then, how intimately associated are the motor regions of the brain and the muscles; when the latter are braced up, the former are active, and there is a corresponding condition between the laxity of the one and the inactivity of the other. Not only is the connection of the nerve-centres in the spinal cord necessary for the nutrition of the muscles and accumulation of energy, but it seems as if the higher centres must be in action for the continued preservation of their tension. Let the activity of the former for a moment cease, as in sleep; the muscles become relaxed, the head drops on one side, the mouth opens, and if the individual be standing, he falls to the ground. There seems to be a constant need of action in the higher motor cells to preserve the body in position, for between contraction of the muscles on the one hand, and complete relaxation on the other, there is an intermediate condition of tension necessary for this preservation of the balance of the body ; there must therefore be a state of nerve centres both of cord and brain, which is one neither of great energy nor of repose, but one of contiauous action. 
If we have a patient who is subject to falling, it is evident that the higher motor regions, or some part of the lower track leading to the muscles, has for the moment become inert; and we ask ourselves in what way has this been brought about. One of the best known facts in reference to the physiology of the brain is, that its function is dependent directly upon a due supply of blood; not only is the latter necessary for its nutrition, but for its momentary activity. If the circulation be arrested, unconsciousness occurs instantly, and the person falls. It is evident that a general cessation of the circulation would result not only in unconsciousness, but in a loss of other functions of the organ; it does not, however, follow that there might not be an arrest to the flow of blood in some portion of the brain, leading to a paralysis of certain muscles without loss of consciousness, and no doubt this does often happen in hysterical women. The sudden falling in syncope appears clearly to arise from an arrest of the flow of blood to the brain, as the patient becomes pale and pulseless, and the local effects of arrest are seen in the case of hemiplegia, resulting from ligature of the carotid. As it is important to bear these great facts in mind, it may be as well to refer to the experiments of Sir A. Cooper, performed more than fifty years ago. ${ }^{1}$ He commenced by tying the cerebral vessels in dogs, and observing the effects. He first ligatured the carotids, which produced some temporary disturbance in the respiration and circulation. As soon as the animal was restored, he exposed the vertebral arteries and compressed them; the dog immediately became unconscious and seemed to be dead; he removed the pressure, and the animal as speedily recovered. He compressed the vessels a second time, and then, as before, there followed loss of motion and apparent death; when the ligature was loosened, the animal came to, with a deep inspiration. After some hours he compressed the vessels a third time, and repeated it in all six times, and always with exactly the same result. Sir Astley observed that this compression of the vertebrals after the carotids were tied put an entire stop to the functions of the brain. He then took another dog, and tied the vertebrals; this produced a greater 1 'Guy's Hospital Reports,' vol. i. 1836. 
effect, as it seemed to paralyse the forelegs. When on the following day the animal had recovered, he compressed the carotids, immediately it fell, losing all sensation and volition; on removing the pressure it soon recovered. He performed this experiment four times with the same result. He then took another animal, and tied all the four vessels; it ceased to breathe, and soon died. Sir A. Cooper adds, "this is a most decisive experiment, showing the effect of the arrest of blood in the vessels of the brain in stopping respiration, volition and sensation, the result being striking and immediate." Sir Astley then tried the effects of pressure on the neck, with the same results, or in his own words: "The same effect of interrupting the stream in the vertebrals and carotids was produced in an equally conspicuous manner without the application of ligatures, as follows: The animal was held in a convenient position, with its neck extended and its head thrown back. I then applied my thumbs so as to compress at the same time the two vessels on each side, taking care to leave the trachea entirely free from compression. Respiration ceased in a few seconds; some struggling then took place, and the animal appeared dead. The pressure being then removed, the respiration was completely suspended; but artificial motion being given to the ribs, the animal gasped, began to breathe quickly and recovered. I also put a ligature around the neck, close to the sternum, so as to compress the carotid and vertebral arteries, but the trachea was excluded by passing the ligature behind it. Although the trachea remained free, as soon as the ligature compressed the carotid and vertebral arteries, breathing ceased, and all the functions of the brain were destroyed."

Not being quite sure that he might not have injured the nerves, he proceeded to put ligatures on the pneumogastric, phrenic and sympathetic nerves, or in his own words: "Before I would venture to draw conclusions from the experiments above detailed, I was desirous of convincing myself that no injury done to the nerves could have influenced in any material degree effects which had been observed. I proceeded therefore to investigate the consequences of applying ligatures to the principal nerves of the neck."

vol. IX. 
Sir A. gives the results of these experiments, which were negative as regards the object he had in view, and concludes his paper with these words: "The sudden death that takes place from pressure at the sides of the neck must not be attributed to an injury to the nerves, but it is owing to the impediment to the due supply of blood to the grand centre of nervous influence."

With our improved experimental methods I do not know that physiologists would be altugether satisfied with Sir A. Cooper's conclusions, and more especially as it is now generally believed that an inhibitory action can be produced on the different organs of the body through the nervous system, and that the brain can be included in the general law; indeed it is probable that cerebrum, cerebellum and spinal cord may possess some inhibitory power over one another. It may be a question, therefore, whether some direct influence may not be exerted through the nerves, either on the heart or the brain, in such an experiment as that made by Sir A. Cooper by pressure on the neck. Dr. de Watteville informs me that he has often performed the same experiment on the human subject with like results : by placing his thumbs on each side of the neck, and exerting a little pressure, he can make the strongest man fall at his feet. He believes that he compresses the vagus, and that it is through this nerve that the effect is produced. Czermak, it may be remembered, was in the habit of showing how by pressure on this nerve he could influence the action of his own heart. These opinions, it is seen, are in favour of the brain being influenced through the heart.

I have long known, and been in the habit of teaching as an important practical fact, that pressure on the neck, as in hanging or garotting, will often produce instantaneous insensibility and death ; and that strangulation by no means implies congestion of the lungs and engorgement of the right side of the heart, as writers on medical jurisprudence have maintained. The fact is of the same nature as that exhibited in Sir A. Cooper's experiment. Even death by obstruction of the larynx is sometimes instantaneous, and not accompanied by the usual symptoms of suffocation, as if there had occurred a sudden paralysis of the respiratory centre. 
That the brain can be acted on directly independent of the heart would seem to be proved by cases of shock, whether this be physical or moral. A blow on the head of a man at once "makes havoc among those tender cells, and checks his power to shape," and at the same time produces a paralysis of motion and of all the senses. A fright will produce exactly the same result, as $I$ have witnessed on several occasions in the cases of women who have lain for days in a state of torpor or trance after some dreadful catastrophe. When under similar conditions the patient has been paralysed on one side only and retained her consciousness, we cannot but believe that one half of the brain has been inhibited in action, as in hysterical hemianæsthesia. If the same result should follow an injury to the head, as I have on several occasions seen, it would follow that a blow might be sometimes given with that exact amount of force which would concuss one hemisphere of the brain and not the other. All these cases show that the brain can receive a direct influence through the nervous system without introducing the instrumentality of the heart's action. Nevertheless the functionising power of the brain does seem to be momentarily dependent upon the heart. If this organ stops, the individual is dead; a blow on the abdomen has been known to arrest suddenly the heart's action, and death is instantaneous. Were it not so, wo should have to believe the ghastly French stories of guillotined heads talking and grimacing. In favour of direct inhibition of the brain through nervous influence, $I$ might mention the case of wild animals suddenly expiring from fright, and the lesser effects produced upon them by the so-called mesmeric process, whereby they will lie for a long time in a completely torpid state. A sudden strange noise will cause my parrot to drop from its perch as if it had been shot; an inhibitory action on the brain through the auditory nerve. I have purposely avoided speculating upon the supposed inhibitory action of the brain on the cerebellum or the spinal cord, as the question has not been satisfactorily determined, being content rather to speak of the clinical facts which one is anxious to elucidate. These seem rather to show that the activity of the cord is not only associated with, but dependent upon that of the brain. 
All the instances mentioned show how directly the healthy working of the muscles is under the influence of the brain, and that the activites of the two structures correspond. In sleep, the brain is inactive and the muscle relaxes; in close attention, the muscle contracts; and in a determined state of mind, as in unger, the muscles would be tightly knit up. Just as the passions of the mind vary in kind and degree, so $I$ apprehend the state of the muscles varies, and similar degrees of contraction occur under simple mechanical conditions. If, for example, the arm be bent by flexing the muscles of the elbow, it may be retained in that position for a long time without fatigue; but if any weight be placed in the hand, the flexors have to exert a greater power to overcome the strain, and I apprehend the contraction of the muscle in the two cases means two different conditions; there is evidently a greater expenditure of force in the latter case, with a corresponding fatigue; but what the difference is in the anatomical constituents of the muscle I would not venture to say. Since the natural tension of the muscle is only preserved whilst it is attached to a healthy nerve-centre, it follows that its integrity and reinforcement after exertion must depend on nerve influence; but since during the stimulation of the motor nerve it is undergoing exhaustion, it necessarily follows that it is during its relaxation and state of rest the muscle is acquiring new vigour. The energy of the muscle is produced in itself, and is not a direct power sent by the nerve, for if not so, a horse would not be stronger than a man. The muscles are like accumulators in which a chemical force is stored up ready to exhibit itself as electricity, but which can only be produced under the influence of a battery. The subject of degeneration and repair of muscle has been admirably discussed by Dr. Gaskell in a paper in the 'Journal of Physiology.' He maintains that there is good reason to believe that all the muscles are like the heart, innervated by two sets of nerve fibres of an opposite character ; and therefore, as motion tends to wear out. the muscle, so there is an inhibitory nerve which stops all action, and at the same time allows the renovation of the muscle to take place.

All the questions involved in the subject which $I$ have been 
discussing are by no means exhausted, for we have taken no heed of the intermediate apparatus between the motor brainregion and the muscles. Seeing that the fibres of the motor truct proceed mostly to the anterior cornua of the cord, and from these again other nerves pass on which stimulate the muscles, it is probable that nearly all the cerebral influence is conveyed through the spinal system. The size of the cord would be opposed to the idea of a more direct connection, and we know that there is no need of it, seeing that the muscles act in groups under the direction of special centres, as for example in the process of talking, where a large number of muscles innervated by numerous nerves are set in operation through a centre which is regulated by the brain. If this is true, the contrary is true; and when the brain is inert, the spinal centres are at rest. We have nothing to show (except speculatively) the inhibitory action of one on the other. Of course in disease a so-called cerebral and spinal paralysis are very different. Any lesion between the brain and spinal centre, whence proceed nerves to a limb, does not affect in any way the nutrition of the latter; the limb is healthy, but it cannot be reached, as the line of communication between it and the volitional region of the brain is severed. A lesion of a spinal centre, however, or of the nerves proceeding from it, produces loss of nutrition of the muscles and their speedy atrophy. I know nothing of such a result as this arising from a mere inhibition of the function of the cord, but an inhibitory arrest of function of certain parts of the cord must, I apprehend, continually occur. That the cord can be stopped in its ordinary physiological work without any structural change occurring in it, is seen in cases of angular curvature, where a patient may be totally paralysed for many weeks and then perfectly recover. To say that the cord has been pressed upon, does not explain how the pressure has caused it to cease work without any material damage having been inflicted upon it. In the acute ascending, or Landry's paralysis, no change may be found on the minutest examination of the cord.

That the cord may be the seat of an inhibitory influence I infer from the fact, that in some cases of very chronic spine disease, patients have suffered, amongst their earlier symptoms, 
from sudden falling. A young lady, now hopelessly paralysed, used about two years ago to suddenly fall whilst walking; she would then rise and proceed as before. On the closest investigation there did not seem the slightest suspicion of even a momentary loss of consciousness. And in the case of a man who died in the hospital of general degeneration of the cord; his first symptom was falling in the street. In other cases which I have not had an opportunity of following, the result has not been known. I mention in my published lectures, under the heading "transient paralysis," the case of a butcher who whilst carrying weights would often suddenly fall, at other times lose the power of speaking. Without any apparent reason seizures would come on, when his speech suddenly failed, and he was quite unable to move his limbs, swallow, cough or spit. There was no symptom of epilepsy, and no spasm. I also mention the case of a gentleman who would suddenly lose all power of walking, and sometimes would find he was unable to speak. This was not due to spasm, but to relaxation of the muscles, for his jaw would drop, and he was unable to close his mouth. His wife often held up his jaw until power had returned. To use his own expression, everything was going on regularly in his body, when suddenly there was a reversal of the engine. He eventually lost these attacks. I was in the habit of illustrating to students the difference between paralysis arising from organic disease of the cord, and that arising from a mere inhibited action, by taking two watches which were not going. It was impossible to say from looking at the exterior what was the cause of this; but on examining the inside, we found one seriously damaged in its chain or spring, whilst the other was a perfect watch, but had not been wound up. Many slighter forms of transient paralysis one occasionally meets with, as when a patient describes a sudden weakness coming over an arm, and any object which was being held fills to the ground. A gentleman for two years after typhoid fever, although he could walk well, would be occasionally and temporarily seized with aJmost complete paraplegia.

It will be seen that where falling has occurred with loss of consciousness, we have placed the cause in the brain proper; and when there has been no loss of consciousness, we have 
looked for a cause in the motor tracts below, or in the spinal centres; but there seems no reason to doubt the possibility: of the function of certain motor areas being inhibited alone, just as these may be disturbed alone in cases of syphilitic disease accompanied by hemi-convulsion without loss of consciousness. As I have before said, I believe the hysterical hemianæesthesia is due to an inhibitory influence operating on one hemisphere, for there is not only a paralysis of half the body, but accompanying this there is often an obliquity of the mind. That consciousness remains when a large part of one hemisphere is destroyed has frequently been observed.

Since in sleep there is the same loss of consciousness and paralysis of the muscles as in syncope, I have assumed that the cause is the same in both; a diminished circulation in the brain. This has been proved to demonstration in the case of sleep, and is exactly what might have been imagined by considering the varying function of organs. Sleep, which has been regarded as one of the most mysterious phenomena of the animal economy, is not peculiar to the brain, but is merely the most striking example of the alternating action of the functions of the human body. We have just now seen how the muscular apparatus wears out, and is again replenished by rest; and as for the stomach, it is quite a different organ when in action and at rest. In the latter condition it is small, with its interior pale, containing little blood, and its secretion scarcely acid: the organ is asleep; but immediately food is put in it, it wakes up, blood flows into its structure, an acid secretion is poured out, and it begins to act in a peristaltic manner on the material within.

Readers may be disappointed at my not arriving at definite conclusions on the subject so important and interesting as that which I have been discussing. I am of opinion that the time is yet distant when the questions $I$ have raised can be satisfactorily solved, seeing that they include some of the deepest problems in the physiology of the nervous system. I have merely in a desultory manner spoken of a symptom with which we are constantly meeting, and expressed some thoughts which necessarily arise in considering it. 